\title{
Avaliação da interferência do laser no material biológico vivo
}

\author{
Juliana V. L. Botega ${ }^{1}$, Roberto A. Braga Júnior², Maurício P. P. Machado ${ }^{3}$, Luiz A. Lima² \& Rafael O. Faria ${ }^{4}$
}

\begin{abstract}
RESUMO
Algumas das características especiais do laser associadas a fenômenos ópticos, permitem uma série de aplicações na área biológica, baseadas no fenômeno conhecido como "biospeckle". A técnica do biospeckle tem sido desenvolvida como uma ferramenta para análise da atividade de materiais biológicos, monitoramento de umidade, nível de atividade e presença de parasitos, dentre outros atributos. Apesar do monitoramento ocorrer através da iluminação do material por uma fonte de laser de baixa potência, a influência da luz na atividade biológica do espécime passa a ser sempre uma variável a ser investigada. Com este trabalho se objetivou avaliar a influência do laser na análise da atividade biológica medida pelo biospeckle de um tecido vegetal, caso em que uma folha de planta de café teve, como parâmetro, 0 tempo de iluminação. Utilizaram-se duas abordagens de iluminação, a primeira incidindo só 0 laser na folha e a segunda tendo o laser incidindo com uma fonte de luz não coerente, iluminando 0 ambiente. Pelos resultados experimentais verificou-se que os dados da atividade não exerceram influência significativa com a incidência do laser nem da luz, não coerente ao longo do tempo.
\end{abstract}

Palavras-chave: biospeckle, análise de imagem, café

\section{Evaluation of the influence of laser in live biological materials}

\begin{abstract}
Some of the special characteristics of the laser associated with optical phenomena allows a series of applications in the biological area, based on the well-known phenomenon biospeckle. The technique of biospeckle has been developed as a tool for analysis of biological materials activity for the checking of moisture, level of activity, presence of parasites, among other attributes. Although the monitoring occurs by illuminating the material with a source of low power laser, the influence of the light on the specimen's biological activity becomes al ways a variable to be investigated. This work had as its objective to evaluation the influence the laser in the analysis of the biological activity measured by the biospeckle of a vegetal tissue; in this case a leaf of coffee plant, using the illumination time as parameter. Two ways of illumination had been used, the first one being reflecting only the laser on the leaf; and the second one the laser reflecting with a source of non-coherent light illuminating the environment. From the experimental results, it was verified that the data of the activity did not have any significant influence with the incidence of the laser, as well as of the non-coherent light throughout the time.
\end{abstract}

Key words: bio-speckle, image analysis, coffee

\footnotetext{
${ }_{1}^{1}$ Doutoranda do PPG EA/UFLA. Fone: (35) 9943-6457. E-mail: julourenconi@tpnet.psi.br

2DEG/UFLA. Fone (35) 3829-1362. E-mail: robbraga@ufla.br; Ialima@ufla.br

${ }^{3}$ Bolsista do CN Pq/PIBIT, Engenharia Agrícola UFLA. Fone (35)3829-1362. E-mail: mauricio.ufla@gmail.com

${ }^{4}$ Bolsista do PIBIC/FAPEMIG, EA/UFLA. Fone (35)3829-1362. E-mail: rafael.ufla@gmail.com
} 


\section{INTRODUÇÃO}

A introdução do speckle dinâmico como fenômeno capaz de mensurar a atividade biológica em tecidos animais e vegetais, tem sofrido grande avanço, desde a década de 1970, quando Briers (1975) propôs a medição da microcirculação do sangue em tecido humano.

Através dos trabalhos que se seguiram, buscou-se dominar a técnica, além de descobrir novas aplicações para o uso do laser e de desenvolvimento de métodos de análise do speckle dinâmico, como instrumento de medição da atividade biológica como, por exemplo, os trabalhos de Fujii et al. (1985), Oulamara et al. (1989), Aizu \& Asakura (1991), Arizaga et al. (1999) dentre outros trabalhos.

$\mathrm{Na}$ agricultura, o uso de um laser de baixa potência e o fenômeno produzido por ele, o biospeckle, foram a base para muitas aplicações, considerando-se sempre a não influência desta luz coerente nos níveis de atividade do tecido em análise. Trabalhos para monitorar a atividade da semente (Braga Jr. et al., 2003), a presença de fungos em sementes (Braga Jr. et al., 2005), danos em frutos (Pajuelo et al., 2003), monitoramento da qualidade de laranja (Rabelo, 2000) e maturação de laranjas (Rabelo et al., 2005) podem ser exemplos do uso desta metodologia sempre com a consideração da não influência do laser nos resultados.

O laser pode também ser utilizado como ferramenta de intervenção e provocação do tecido iluminado. Os trabalhos de Vasilenko (1992), Toth et al. (1993), Drozd (1994) e Dudin et al. (1995) mostraram a viabilidade de uso desta luz como agente capaz de influenciar o metabolismo de sementes. A potência do laser passa a ser o diferencial de sua aplicação, podendo ser instrumento de análise ou ferramenta de ativação do metabolismo e intervenção no tecido.

Apesar de trabalhos como o de Seitz (1979), por exemplo, que elimina a influência de algumas faixas de frequiência na velocidade das correntes citoplasmáticas ou na atividade dos cloroplastos, além do uso de baixas potências nas iluminações, não é possível eliminar os freqüentes questionamentos da influência do laser na atividade dos materiais biológicos, uma vez que a luz é conhecida, em especial para os tecidos vegetais, como promotora do metabolismo.

Neste trabalho se avaliou a influência do laser na atividade biológica de um tecido vegetal tendo como atributo o tempo de iluminação. De forma específica, folhas de uma planta de coffea arabica foram iluminadas e sua atividade acompanhada pelo fenômeno do biospeckle.

\section{MATERIAL E MÉTODOS}

No experimento se utilizaram: um microcomputador, aplicativos computacionais para processamento de imagens como o $\mathrm{Scilab}^{\mathrm{TM}}$ e o ImageJ 1.37, uma fonte de laser HeNe $10 \mathrm{mw}$ e comprimento de onda de 632,8 nm, uma câmara CCD ("Charge Coupled Device") de 640 x 480 pixels e uma muda de Coffea arabica, como mostrado esquematicamente na Figura 1, além de duas abordagens, em que a primeira adota o laser como a única fonte de iluminação direcionada na folha e, na segun-

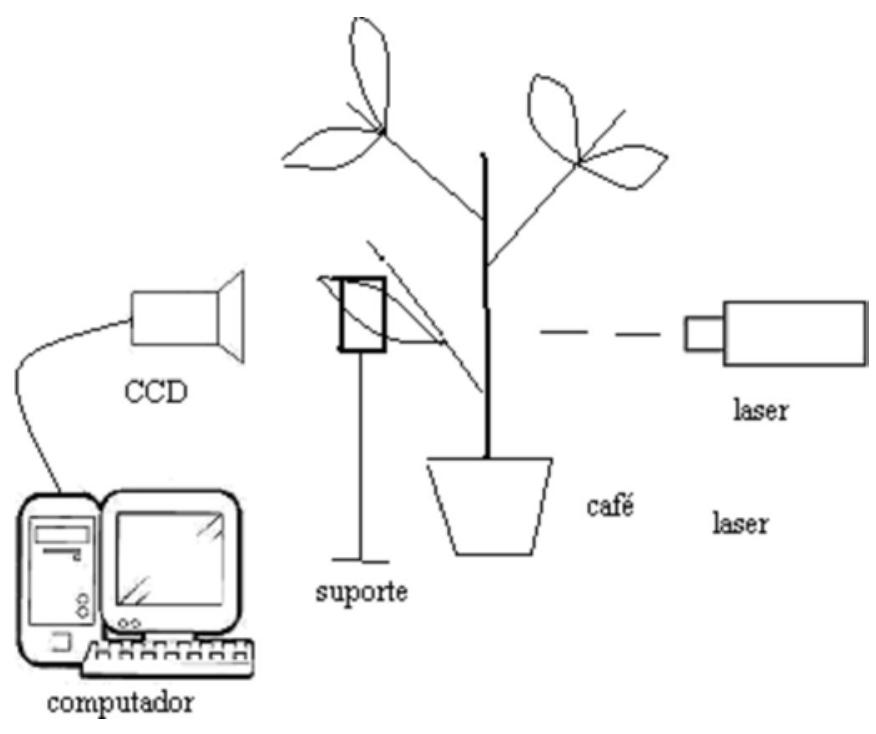

Figura 1. Arranjo experimental para iluminação da folha de café

da, são utilizados o laser e uma fonte de luz não coerente, que ilumina todo o ambiente. A fonte de luz não coerente utilizada foi do tipo fluorescente com potência total de 160 W. Obter-se-á, com processamento, uma imagem que representa a atividade biológica proporcional ao nível de cinza; desta forma e se houver uma área com um nível de cinza mais elevado, ou seja, mais clara, isto estará relacionado à alta atividade do material neste ponto. As configurações experimentais dessas duas abordagens se diferenciaram na presença ou ausência de luz não coerente no laboratório e no processamento de imagens.

\section{Aquisição de imagens}

Selecionou-se uma folha do cafeeiro, a qual foi fixada a um suporte, sem ser retirada da planta. Uma face da folha foi iluminada com o laser e na face oposta se colocou a câmera CCD, que capturou as imagens, formando os padrões do speckle. As imagens foram processadas pelo método proposto por Fujii et al. (1985).

O laser foi ligado uma hora antes do início da iluminação. Utilizaram-se 20 pontos de análise no tempo; ressalta-se que o primeiro ponto estava com um intervalo de 30 s do início da iluminação e, a partir deste ponto até o décimo sexto ponto, utilizaram-se intervalos de 3 min entre cada avaliação; do décimo sétimo até o vigésimo ponto, os intervalos foram de 15 min, totalizando 105 min de iluminação; para cada ponto foram capturadas 100 imagens que, processadas pelo método de Fujii et al. (1985), estavam prontas para a etapa de processamento das imagens de atividade.

\section{Processamento de imagens}

Utilizaram-se: utilizado o método do somatório dos níveis de cinza das imagens provenientes do processamento pelo método de Fujii, através do software Scilab $4.0^{\mathrm{TM}}$ e o método do cálculo de área do speckle, pelo software ImageJ1.37, para processar as vinte imagens de cada etapa.

Através do SIP, toolbox instalado no Scilab $4.0^{\mathrm{TM}}$, foi possível o cálculo do somatório dos níveis de cinza nas imagens 
com extensão de arquivo.BMP por meio da função gray_imread.

Para o cálculo da área do speckle através da a abordagem laser com a luz não coerente, usou-se a seguinte estratégia: transformação da imagem para 8 bits, utilização da função Find Edjes, proporcionando bom contraste das bordas do speckle, binarização e uso da função Dilate; já no cálculo da área de atividade em que se usou a abordagem sem luz coerente, lançou-se mão apenas da ferramenta binary, que limiariza a imagem, segmentando-a do fundo e permitindo o cálculo da área no ambiente do ImageJ1.37.

\section{RESULTADOS E DISCUSSÃO}

\section{Processamento de imagens}

\section{Primeira abordagem sem a adição da luz não coerente}

O resultado obtido é mostrado na Figura 2, na qual a sequiência de imagens mostra os passos para seleção da área a ser mensurada. Na Figura 2A é possível observar a imagem no formato. BMP obtida pelo método de Fujii et al. (1985) e, na Figura 2B, a binarização.

A.

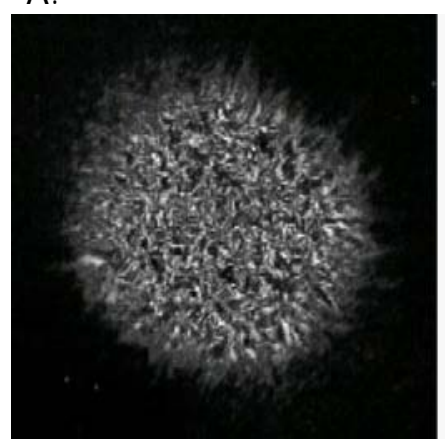

B.

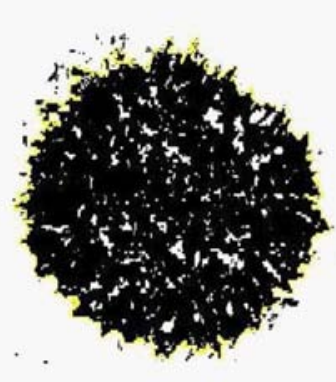

Figura 2. Etapas de visualização da imagem no formato .BMP e binarização

Segunda abordagem com a adição da luz não coerente

$O$ resultado obtido para este processamento pode ser observado na Figura 3, que apresenta a imagem no formato BMP obtida pelo método de Fujii e os passos de utilização da função Find Edjes, binarização e uso da função Dilate, respectivamente.

Nesta etapa e por conta da luz ambiente reduzir o contraste do laser na folha, foram necessários mais passos para a obtenção da área.

\section{Análise do nível de cinza e da área, ao longo do tempo nas duas abordagens}

Percebeu-se que não só a área do speckle, mas também os níveis de cinza mostraram tendência a manter seus valores constantes. O cômputo da área foi o que melhor representou esta tendência com a menor variação de valores; laser e luz não coerente: Área $1.07 \%$ e níveis de cinza 6.45\%; laser: Área $1.98 \%$ e níveis de cinza $3,56 \%$.

As Figuras 4A e 4B apresentam as abordagens de medida pelo somatório de cinza e pela área da iluminação utilizando, respectivamente, a não injeção de luz não-coerente e injeção, além do laser.

A injeção de luz não-corente ocasionou dois fenômenos nos gráficos de tendência que mostram a influência desta luz no cálculo, utilizando a somatória dos níveis de cinza (Figura 4B); o primeiro fenômeno foi o aumento do patamar da curva de tendência quando mais luz atingia o objeto, enquanto o segundo foi relativo ao aumento da atividade da folha expresso pelo nível de cinza, evidenciando a influência de luz na folha nos primeiros momentos de iluminação.

Após iluminação este acréscimo pôde ser observado quando apenas o laser incidia em parte da folha pelo cômputo da área; este fenômeno ocorreu em apenas um ponto inicial, ocasionando um acréscimo de atividade medida em 1\% (Figura 4A).

Os resultados mostram que a incidência do laser não afeta significativamente a atividade das folhas, confirmando experimentos de Seizt (1979), e os de Rabelo (2000) que avaliou a influência do laser no metabolismo de laranjas, evidenciando que o tecido sujeito a um laser de baixa potência não alterou os resultados do biospeckle no tempo.

A iluminação pelo laser em ambiente com a presença de luz natural ou artificial não-coerente, não afeta o resultado em especial se o cômputo avaliar a área de atividade, caso em que se deu redução dos valores das áreas, causada pela maior dificuldade de segmentação.

Ressalta-se que a influência do laser de baixa potência em outros materiais deve ser avaliada, uma vez que não se pode estender os resultados conseguidos pela folha se considerar aspectos como a fotofobia de algumas espécimes ou a fotoativação de espécimes, mesmo com pouca luz.

C.

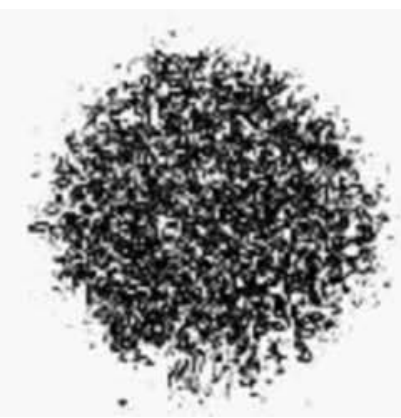

D.

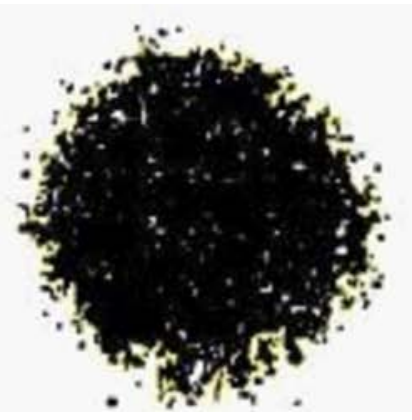

Figura 3. Etapas de transformação da imagem (A) após Fujii, (B) após função Find Edjes, (C) binarização e (D) uso da função Dilate 


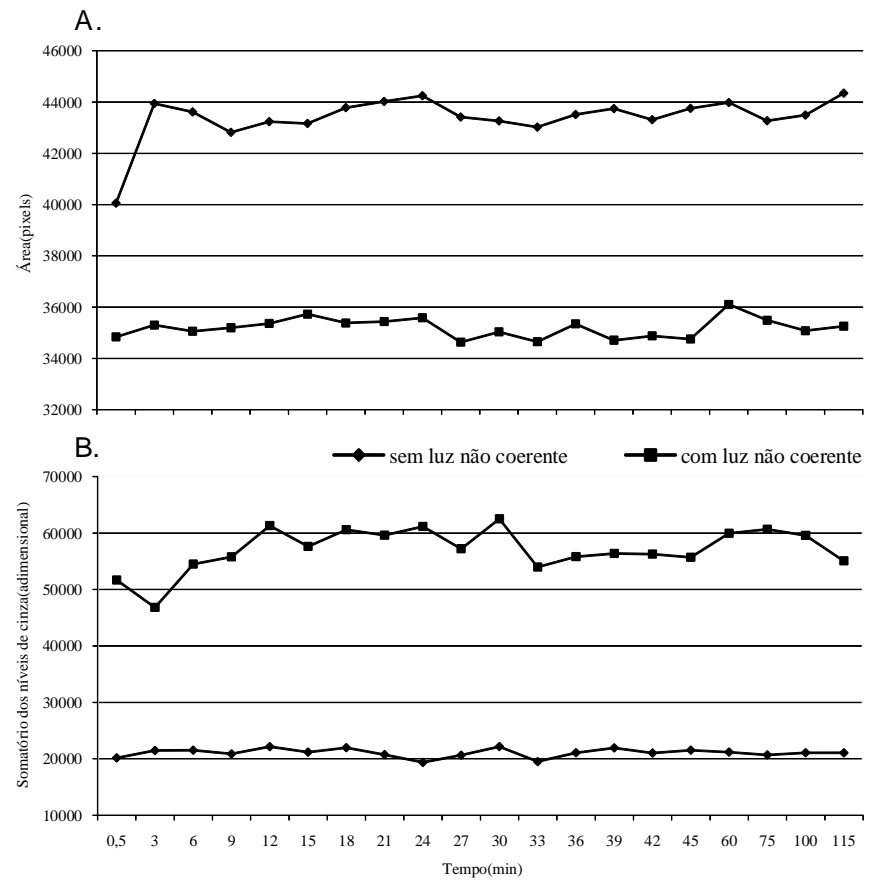

Figura 4. G ráfico de comparação de área ao longo do tempo, com e sem adição de luz coerente $(A)$, gráfico de comparação de níveis de cinza e área, ao longo do tempo com e sem adição de luz coerente (B)

Esses resultados com a folha permitem trabalhos de análise de sua atividade usando-se o laser sem preocupação com a atividade biológica e na presença de luz não coerente.

\section{CONCLUSÕES}

1. Conclui-se que o cálculo da área e o somatório dos níveis de cinza não tiveram influência significativa pela incidência do laser, ao longo do tempo em material vivo.

2. A luz não coerente não interfere nos resultados de cálculo de área nem nos níveis de cinza.

3. Os resultados do cálculo da área tiveram menor coeficiente de variação em relação aos de somatório de nível de cinza.

\section{AGRADECIMENTOS}

À Universidade Federal de Lavras, Capes, CNPq e FAPEMIG.

\section{LITERATURA CITADA}

Aizu,Y.; Asakura, T. Bio-speckle phenomena and their application to the evaluation of blood flow. Optics \& Laser Technology, v.23, n.4, p.205-219, 1991.

Arizaga, R.; Cap, N.; Rabal, H.; Trivi, M. Activity image in dynamical speckle. In: Guzman, A. (ed.). Third Iberoamerican Optics Meeting, Proceeding SPIE 3572, 1999. p.310-314.

Braga Jr., R. A.; Fabbro, I. M. , Borem F. M., Rabelo, G. F.; Arizaga H.; Rabal, J.; Trivi. M. Assessment of seed viability by laser speckle techniques. Biosystems Engineering, v.86, n.3, p.287-294, 2003.

Braga Jr., R. A.; Rabelo, G. F.; Granato, L. R.; Santos, E. F.; Machado, J. C.; Arizaga, R.; Rabal, H. J.; Trivi, M. Detection of fungi in beans by the laser biospeckle technique. Biosystems Engineering, v.91, n.4, p.465-469, 2005.

Briers, J. D. Wavelength dependence of intensity fluctuations in laser speckle patterns from biological specimen. Optics Communications, v.13, p.324-326, 1975.

Drozd, D. The effect of laser radiation on spring wheat properties. International Agrophysics, v.8, n.2, p.209-213,1994.

Dudin, G. D.; Loginov, D. A.; Krivosheina, O. S. Irradiation of barley seeds with laser and red light. Zemledelie, v.1, n.1, p.33-53, 1995.

Fujii, H.; Asakvrat,T.; Nohira, K.. Blood-flow observed by time-varing laser speckle. Applied Optics, v.10, n.3, p.104-106, 1985.

Oulamara, A.; Tribillon, G.; Duvernoy, J. Biological activity measurement on botanical specimen surface using temporal decorrelation effect of laser speckle, Journal of Modern Optics, v.36, n.2, p.165-179, 1989.

Pajuelo, M.; Baldwin, G.; Rabal, H.; Cap, N.; Arizaga, R.; Trivi, M. Bio-speckle assessment of bruising in fruits. Optics and Laser in Engineering, v.40, n.1/2, p.13-24, 2003.

Rabelo, G. F. Avaliação da aplicação do speckle dinâmico no monitoramento da qualidade da laranja. Campinas: UNICAMP, 2000. 149p. Tese Doutorado

Rabelo, G. F.; Braga Jr., R. A.; Fabbro, I. M. D. Laser speckle techniques in quality evaluation of orange fruits. Revista Brasileira de Engenharia Agrícola e Ambiental, v.9, n.4, p.570-575, 2005.

Seitz, K. Cytoplasmic streaming and cyclosis of chloroplasts. In: Haupt, W.; Felnleib, M. E.(ed.). Encyclopedia of plant physiology, new ser. Berlin: Springer-Verlag, 1979. p.150-169.

Toth, M.; Kerpesi, I.; Kozma, L.; Klujber, L. Influence of wavelength laser lights on the carbohydrate metabolismin germinating maize seeds. Acta Botanica Hungarica, v.38, n.1, p.421-430, 1993.

Vasilenko, A. V. Accelerating the growth of scots pine seedlings. Lesnoe Khozyaistvo, v.3, n.1, p.39-40, 1992. 